\title{
Medical Education and Mental Wellbeing During COVID-19: a Student's Perspective
}

\author{
Stephanie Hiu Ling Poon ${ }^{1} \cdot$ Mathew S. C. Chow ${ }^{1}$ (1) - Wendy W. T. Lam ${ }^{2}$
}

Accepted: 22 March 2021 / Published online: 31 March 2021

(C) International Association of Medical Science Educators 2021

Many countries in the Asia-Pacific were first to experience the wide-reaching impact of COVID-19. It challenged even the most well-endured socio-economic systems, and medical education systems were not exempted from these repercussions, as suspension of physical classes and clinical teachings have forced medical students to adapt to a 'new normal'. A sudden hiatus from traditional in-person teachings was paired with a concomitant rise in the use of online teaching methods and a substantial restriction of clinical learning experiences.

It, therefore, is no surprise that COVID-19-induced changes to medical teachings may influence the psychological wellbeing of medical students. Medical students may find it increasingly challenging to learn, as attending online synchronous or asynchronous didactic lectures, real-time small-group discussions and communication skills teaching have become the norm [1]. Since students are not able to interact with study peers like they did face-to-face, this may give the impression that medical school is a lonely battle. This immense stress could lead to further deterioration of mental wellbeing. Moreover, as clinical judgement is fostered with the gradual accumulation of bedside experience [2], medical students have increasing concerns surrounding inadequate patient exposure and practice opportunities before sitting their final examinations and independently caring for patients [3]. Feelings of helplessness and despair may result from perceived shortcomings in competencies and skills, which were best learnt from traditional tried-and-tested approaches within their medical school and locality. Though

Stephanie Hiu Ling Poon and Mathew S.C. Chow contributed equally to this work.

Mathew S. C. Chow

mathec@hku.hk

1 Li Ka Shing, Faculty of Medicine, The University of Hong Kong, Hong Kong, China

2 School of Public Health, Li Ka Shing, Faculty of Medicine, The University of Hong Kong, Hong Kong, China some medical schools have started to trial telemedicine for educational purposes [4], technological and logistical limitations hinder the feasibility and effectiveness of such efforts [5], particularly in low-resource settings. This is exacerbated by pre-existing concerns about patient privacy and confidentiality. With medical students joining the health workforce prematurely in many countries to fight the pandemic, these worries have become more relevant.

As medical students in Hong Kong, we were also not exempt from adapting to these pandemic-induced changes. As international students travelled back to their respective home countries, time differences, inevitably, impeded meaningful participation in scheduled online classes [6]. Moreover, our exchange programmes and electives with medical schools and research institutions abroad had to be cut short or cancelled entirely. Our clinical teachings were continuously postponed as daily incident COVID-19 cases spiked. Bedsides, teachings were enabled via telepresence for clinic consultations and virtual ward rounds to facilitate continuous learning during the pandemic, yet most students perceived them to be less genuine than being able to perform physical examinations on patients in person $[7,8]$. Infection control measures were implemented at academic testing sites. The fear of recording a slightly elevated body temperature resulting in being banned from entering testing venues caused anxiety in students who already had continuously delayed examinations. For some of us undertaking intercalated research degrees, laboratory-based projects were fortunately still allowed to be carried out but with strict infection control measures in place. Although the option to stay at home during the pandemic was offered to students, most of us continued commuting back to the laboratory daily despite the rising numbers in COVID cases, due to concerns over our work progress as well as supervisors' expectations. Others involved in clinical research modules were not allowed hospital access due to infection control measures. Having already limited time to complete their research, their psychological burdens 
were further exacerbated by the current inevitable delays in carrying out their work, as well as fear of their future workload when they have to catch up with their research progress whilst managing their academic studies during their final years of clinical years.

Our personal experience coping both academically and mentally with abrupt changes to learning formats during the numerous waves of COVID-19 outbreak within the past months prompted us to investigate how other medical students across Asia cope with such challenges.

After obtaining ethical approval, we distributed an online questionnaire to investigate the psychological wellbeing of 374 Asian medical students (from Afghanistan; Australia; China, including Hong Kong and Taiwan; India; Indonesia; Japan; Kyrgyzstan; Malaysia; Nepal; Pakistan; the Philippines; South Korea; Singapore; Thailand and Uzbekistan) in May 2020 during the COVID-19 pandemic. The Patient Health Questionnaire-9 (PHQ-9), WHO-5 Well-Being Index, General Anxiety Disorder-7 (GAD-7) and K-6 were adopted for evaluation of depressive symptoms, wellbeing, anxiety and psychological distress respectively. A total of $37.7 \%$ of respondents had a PHQ-9 score $\geq 10$ out of 27 , higher than the pooled global depression prevalence of $27.2 \%$ [9] and $28.0 \%$ [10] found previously. Based on the WHO-5 Well-Being Index, a majority $(59.1 \%)$ reported a score below the passing mark of 52 out of 100 , whereas only $7.2 \%$ had good wellbeing status $(\geq 72)$. A total of $30.4 \%$ had probable anxiety (GAD-7 score $\geq 10$ ), whilst $18.7 \%$ had probable serious mental illness (K6 score $\geq 19$ ). We also asked participants about satisfaction towards their respective medical schools' adaptations to teaching arrangements. Technology use and access to stable internet connection were major themes that emerged with regard to online learning. This is particularly a challenge for students studying in less-resourced settings. Variations in curfew periods across the country meant students had to travel across provinces to cater to their exam schedules if they relied on external sources of internet connection outside their home. While didactic lectures and small group tutorials were more easily converted to a virtual format [11], teaching moments that require more specialised devices and arrangements, such as bedside teaching with real patients, necessitated longer periods of adaptation, particularly in schools that did not originally have a remote teaching structure in place. Moreover, training of educators in navigating virtual platforms was emphasized-specifically, a participant from Manila stated that 'teachers were not given adequate training for conducting online classes, resulting in suboptimal transitioning of teaching format.' All of this significantly adds to their psychological burden. On the other hand, teaching schedule adaptations led to substantial changes and cancellations of student electives and hospital placements, particularly given widespread travel restrictions. A recent UK-based national survey found similar contributing factors towards a deteriorated level of confidence in medical students [12].

Qualitative data revealed that medical students' suboptimal wellbeing stemmed from needing to adapt to a completely new format of learning and assessment. This is coupled with fear and uncertainty towards their future, as well as concerns of expectations from teachers, families and peers as they proceeded towards their final stages of medical education. Moreover, some students felt that institutional support was not sufficient to help them transition across learning modes, whereas others believed inadequate communication regarding the restructuring of their teaching schedules and examinations exacerbated their worries. Substantial and swift communication between students, medical school administrators and teachers reduced medical students' mental distress. Though the surveyed medical students came from different socio-economic backgrounds and received variants of tried-and-tested medical education curricula, common themes emerged both in terms of how medical educators adapted their delivery of effective clinical skills education, and the challenges of how students had to comply and adjust to these alternative teaching methods.

What the current pandemic has induced is not merely a deviation from the norm but a complete departure from established practices and expectations within the medical education system. Mental health support infrastructure, if already existing in medical schools, were compromised and necessitated online consultations with psychological counsellors to care for those in need, particularly during times of uncertainty and volatility. Other than providing post hoc therapy-oriented services and adjuvant wellness programs, curricular reform has been suggested to effectively address long-standing mental health issues amongst the medical student body. With medical education obliged to adapt to this pandemic, now is the quintessential moment to implement these changes to address root causes of distress while organically fostering resilience skills to better cope with mental health stressors [13]. Student-faculty partnerships, in which student opinions are not only listened to but acted upon, should be promoted. While it may be easier to implement top-down changes on how medical students are taught, transitions to contemporary student centred approaches can maximise effectiveness and student wellbeing during trying times. Research efforts that explore the psychosocial wellbeing of medical students and fresh graduates during these unprecedented times, such as Oxford University's SPICE19 study [14], should be welcomed to formally substantiate these impacts to drive more evidence-based strategies in our approach in supporting medical students, both academically and psychologically.

As the resilience of medical education systems is put to the test, so is the resilience of medical students. In a period of rapid and uncertain change, the mental health of future 
health care professionals should not be put on the sidelines. Our decisions will set precedence for how we act and react during future emergencies that similarly challenge the status quo. Transformations and innovations of current medical education systems due to the COVID-19 pandemic should be leveraged for pedagogical and andragogical advancements to empower and train competent doctors. From the learner side, we believe that medical education should not only train our clinical skills set and competencies but should also foster psychological resilience that will help us navigate through current and future challenges. We believe that the perspectives of students, as the recipients of medical education, should not be overlooked, and that through regular and meaningful communication between students and educators, COVID-adapted teaching methods can be further modified to achieve a compromise between learners' needs and wellbeing and maintenance of the quality of teaching.

Acknowledgements The authors would like to thank Dr. Julie Liao for her guidance in statistical sample size calculation.

Author Contribution SHLP, MSCC, WWTL contributed to the conception and design of the study. SHLP and MSCC contributed to the acquisition of data, analysis and interpretation of data. SHLP, MSCC and WWTL drafted and revised the article critically for important intellectual content and approved of the final version of the manuscript for publication.

Availability of Data and Material Data that supports the findings of this study is available from the corresponding author, MSCC, upon reasonable request.

\section{Declarations}

Ethical Approval Ethical approval for the study was obtained from the Institutional Review Board of The University of Hong Kong/Hospital Authority Hong Kong West Cluster (HKU/HA HKW IRB).

Informed Consent Informed consent was obtained from all participants included in the study.

\section{References}

1. Jumreornvong O, Yang E, Race J, Appel J. Telemedicine and medical education in the age of COVID-19. Acad Med. 2020;95(12):1838-43.
2. Theoret C, Ming X. Our education, our concerns: the impact on medical student education of COVID-19. Med Educ. 2020;54:591-2.

3. Lucey CR, Johnston SC. The transformational effects of COVID19 on medical education. JAMA. 2020;324(11):1033.

4. Dodenhoff KA, Olszewski C, Thomson S. Connecting through the screen: remote learning and telehealth during the COVID-19 pandemic. Acad Med. 2021 (published ahead of print).

5. Iancu AM, Kemp MT, Alam HB. Unmuting medical students' education: utilizing telemedicine during the COVID-19 pandemic and beyond. J Med Internet Res. 2020;22(7):e19667.

6. Rose S. Medical student education in the time of COVID- 19. JAMA. 2020;323:2131-2.

7. Ferrel MN, Ryan JJ. The impact of COVID-19 on medical education. Cureus. 2020;12.

8. Goh PS, Sandars J. A vision of the use of technology in medical education after the COVID-19 pandemic. MedEdPublish. 2020;9.

9. Rotenstein LS, Ramos MA, Torre M, et al. Prevalence of depression, depressive symptoms, and suicidal ideation among medical students. JAMA. 2016;316(21):2214.

10. Puthran R, Zhang MWB, Tam WW, et al. Prevalence of depression amongst medical students: a meta-analysis. Med Educ. 2016;50(4):456-68.

11. Remtulla R. The present and future applications of technology in adapting medical education amidst the COVID-19 pandemic. JMIR Med Educ. 2020;6(e20190).

12. Choi B, Jegatheeswaran L, Minocha A, et al. The impact of the COVID-19 pandemic on final year medical students in the United Kingdom: a national survey. BMC Med Educ. 2020;20(1):206.

13. Slavin SJ, Schindler DL, Chibnall JT. Medical Student Mental Health 3.0: improving student wellness through curricular change. Acad Med. 2014;89(4):573-577.

14. University of Oxford Medical Sciences Division. The Social and Psychological Impact of COVID-19 on Medical Students: NIHR oxford health biomedical research centre news; 2020 [cited 2020 September 19]. Available from: https://www.psych.ox.ac.uk/news/ the-social-and-psychological-impact-of-covid-19-on-medicalstudents.

Publisher's Note Springer Nature remains neutral with regard to jurisdictional claims in published maps and institutional affiliations. 